ARTICLE

https://doi.org/10.1038/s41467-019-11361-z

\title{
Strong stress-composition coupling in lithium alloy nanoparticles
}

Hyeon Kook Seo ${ }^{1}$, Jae Yeol Park (1) ${ }^{1}$, Joon Ha Chang', Kyun Sung Dae ${ }^{1}$, Myoung-Sub Noh ${ }^{2,3}$, Sung-Soo Kim ${ }^{4}$ Chong-Yun Kang ${ }^{2,3}$, Kejie Zhao ${ }^{5}$, Sangtae $\mathrm{Kim}^{3}$ \& Jong Min Yuk ${ }^{1}$

The stress inevitably imposed during electrochemical reactions is expected to fundamentally affect the electrochemistry, phase behavior and morphology of electrodes in service. Here, we show a strong stress-composition coupling in lithium binary alloys during the lithiation of tin-tin oxide core-shell nanoparticles. Using in situ graphene liquid cell electron microscopy imaging, we visualise the generation of a non-uniform composition field in the nanoparticles during lithiation. Stress models based on density functional theory calculations show that the composition gradient is proportional to the applied stress. Based on this coupling, we demonstrate that we can directionally control the lithium distribution by applying different stresses to lithium alloy materials. Our results provide insights into stress-lithium electrochemistry coupling at the nanoscale and suggest potential applications of lithium alloy nanoparticles.

\footnotetext{
${ }^{1}$ Department of Materials Science and Engineering, Korea Advanced Institute of Science and Technology (KAIST), Daejeon 34141, Republic of Korea. ${ }^{2}$ KU-KIST Graduate School of Converging Science and Technology, Korea University, Seoul 02841, Republic of Korea. ${ }^{3}$ Center for Electronic Materials, Korea Institute of Science and Technology (KIST), Seoul 02792, Republic of Korea. ${ }^{4}$ Graduate School of Energy Science and Technology, Chungnam National University, Daejeon 34134, Republic of Korea. ${ }^{5}$ School of Mechanical Engineering, Purdue University, West Lafayette 47907 IN, USA. Correspondence and requests for materials should be addressed to S.K. (email: stkim@kist.re.kr) or to J.M.Y. (email: jongmin.yuk@kaist.ac.kr)
} 
$\mathrm{M}$ echanical stress critically affects various chemical processes ranging from electrochemical energy storage ${ }^{1,2}$ to catalytic reactions $s^{3,4}$ to polymerization ${ }^{5}$. In lithium $(\mathrm{Li})$ insertion electrochemistry, the large stress evolution during lithiation cycles poses major challenges to incorporating alloying anodes in commercialized batteries ${ }^{6,7}$. In addition to causing electrode cracking, stress couples to the insertion electrochemistry, affecting the electrode potential, lithiation kinetics, and Li composition ${ }^{8,9}$. These electrochemistry-stress couplings have been studied in depth, including the stress contribution to chemical potential ${ }^{10}$ or stress-induced kinetic retardation ${ }^{11,12}$. However, how stress solely changes the Li composition in the nanoscale has not been unexplored experimentally ${ }^{13}$, because stress, diffusion, and Li composition are mutually coupled to one another. Understanding the stress-composition coupling would potentially open up various technological applications such as energy harvesters, while providing scientific insights into stress-matter interaction.

While directly observing the composition change under applied stress may shed light on this fundamental question, experimentation has thus far remained difficult ${ }^{1,14}$. To observe the role of stress in battery electrode materials, it is necessary to stress the active material within the iso-chemical potential reservoir (the electrolyte) and simultaneously observe the compositional and morphological changes in situ. Since the compositional spatiodynamics in battery materials occurs on a nanoscale, nanoscale observations are also essential ${ }^{15}$. Realizing this capability has been challenging with the current state-of-theart characterization techniques such as in situ transmission electron microscopy (TEM) on an encapsulated liquid ${ }^{16}$.

Thus, here, we use in situ graphene liquid cell electron microscopy (in situ GLC-EM) ${ }^{17,18}$ to visibly track the temporal phase and morphological evolution of a single nanoparticle while it lithiates under stress. To apply stress in situ, we use tin-tin oxide $\left(\mathrm{Sn}-\mathrm{SnO}_{2}\right)$ core-shell nanoparticles. During lithiation, the oxide shell acts as an effective instrument that applies large hydrostatic compression to the core, while undergoing tensile hoop stress itself. The constant electron irradiation from the TEM column drives the lithiation of the nanoparticles immersed in the liquid specimen, which is a condition similar to the galvanostatic lithiation in a battery cell. $S n$ in tetragonal phase ( $\beta$-Sn, space group $I 4_{1} /$ amd (141), $a=5.83 \AA, c=3.18 \AA$ ) is chosen for its fast Li diffusivity ${ }^{19}$, in efforts to minimize the effect of stress-driven kinetic retardation. Also, owing to its relatively low alloying energy of $-0.48 \mathrm{eV}, \mathrm{Li}_{3.5} \mathrm{Sn}$ is a suitable material for studying the interaction between electrochemistry and stress, which occurs on a comparable energy scale.

\section{Results}

Abnormal phase evolution during shelled particle lithiation. Figure 1 and Supplementary Movie 1 track the phase evolution of a $\mathrm{Sn}-\mathrm{SnO}_{2}$ core-shell nanoparticle during in situ lithiation. The setup for GLC-EM and the lithiation condition inside GLC-EM are described in the Supplementary information (Supplementary Fig. 1, Supplementary Table 1, and Supplementary Notes 1 and 2). The electron diffraction patterns (EDPs) in Fig. 1 reveal that the core-shell nanoparticle lithiates by subsequently nucleating partially lithiated $\mathrm{Sn}\left(\mathrm{Li}_{x} \mathrm{Sn}_{y}\right)$ at $90 \mathrm{~s}$ (Fig. $1 \mathrm{~b}, \mathrm{j}$ ), crystalline $\mathrm{Li}_{7} \mathrm{Sn}_{3}$ at $280 \mathrm{~s}$ (Fig. 1c, k), and $\mathrm{Li}_{7} \mathrm{Sn}_{2}$ phase at $435 \mathrm{~s}$ (Fig. 1d, l). We observe that the EDPs from these phases appear in order, as well as those from $\mathrm{Sn}$ core, $\mathrm{SnO}_{2}$ shell, and $\mathrm{Li}_{2} \mathrm{O}$ generated by $\mathrm{SnO}_{2}$ shell conversion $\left(\mathrm{SnO}_{2}+4 \mathrm{Li} \rightarrow \mathrm{Sn}+2 \mathrm{Li}_{2} \mathrm{O}\right)^{20}$. The $\mathrm{SnO}_{2}$ peaks do not disappear until $280 \mathrm{~s}$, indicating that $\mathrm{SnO}_{2}$ partially remains until $\mathrm{Sn}$ is heavily lithiated. This is surprising since the calculated average potential for $\mathrm{SnO}_{2}$ shell conversion $(1.5 \mathrm{~V})$ is notably higher than that of $\mathrm{Sn}(0.6 \mathrm{~V})$ and suggests kinetically limited $\mathrm{SnO}_{2}$ lithiation ${ }^{21}$. The Sn core could be lithiated first by Li migration through grain boundaries in the $\mathrm{SnO}_{2}$ shell ${ }^{22}$.

Further lithiation, involving significant shell-imposed stress on the core, exhibits unanticipated phase evolution. Instead of forming a fully lithiated $\mathrm{Li}_{22} \mathrm{Sn}_{5}$ phase, the observed $\mathrm{Li}_{7} \mathrm{Sn}_{2}$ phase at $435 \mathrm{~s}$ evolves into $\mathrm{Li}_{x} \mathrm{Sn}_{y}$ or $\mathrm{Li}_{2} \mathrm{Sn}_{5}$ phases (Fig. 1e-g, m,o). These Li-deficient phases are consistently observed, and the final phases observed after $770 \mathrm{~s}$ of lithiation are $\mathrm{Sn}, \mathrm{Li}_{5} \mathrm{Sn}_{2}, \mathrm{Li}_{x} \mathrm{Sn}_{y}$, and $\mathrm{Li}_{2} \mathrm{O}$ (Fig. 1h, p and Supplementary Table 2). The intermediate phases and even pristine $\mathrm{Sn}$ that disappeared during initial lithiation remarkably reappear with the continued lithiation.

These phenomena are consistently observed during galvanostatic lithiation of similar particles in conventional battery cells. For example, in situ small-angle X-ray scattering (SAXS) experiments during lithiation show a sudden increase in Sn content near $\mathrm{Li}_{2} \mathrm{Sn}$ nominal composition (Supplementary Fig. 2). The ex situ X-ray diffraction (XRD) patterns of the fully lithiated core-shell nanoparticles reveal largely Li-deficient phases (Supplementary Fig. 3 and Supplementary Note 3$)^{23}$. During the lithiation of bare Sn particles without oxide shells, however, the Li-deficient phases do not appear. These particles fully lithiate into the $\mathrm{Li}_{22} \mathrm{Sn}_{5}$ phase in the identical GLC setup, clearly indicating the role of the oxide shell (Supplementary Fig. 4 and Supplementary Movie 2).

In situ observation of Kirkendall voiding during lithiation. The abnormal lithiation behavior under stress also accompanies unique morphological evolution, as shown in the time-series TEM images (Fig. 2 and Supplementary Movie 3). The nanoparticle expands upon lithiation and reaches maximum areal expansion of $120 \%$ at $185 \mathrm{~s}$ (Fig. 2a-c, Supplementary Table 3, and Supplementary Notes 4 and 5). As lithiation continues, the nanoparticle briefly fluctuates in size (Fig. 2d, e), followed by spontaneous volume shrinkage and Kirkendall void nucleation inside the core (Fig. 2f, g). The core then wobbles like a liquid, forming a void and a single meniscus at $360 \mathrm{~s}^{21,24-26}$. The core's areal expansion at this point is only $8 \%$, suggesting a large amount of $\mathrm{Li}$ extraction from the core. Indeed, the volume expansion analyses show that the composition in the core changes from $\mathrm{Li}_{3.5} \mathrm{Sn}$ to $\mathrm{Li}_{0.4} \mathrm{Sn}^{27,28}$, corresponding closely to the reappearance of Li-deficient phases in Fig. 1. Such voided morphology also consistently appears in the ex situ observations of galvanostatically cycled particles in the conventional battery setup (Supplementary Figs. 5-7).

The detailed morphology analyses reveal where Li migrates during the core dealloying. Figure $2 \mathrm{k}$ plots the areal change of the cores and average shell thicknesses during lithiation for three different core-shell particles (Supplementary Movies 1, 3, and 4 and Supplementary Figs. 8-10). The shell thicknesses increase during the initial lithiation and soon reach plateaus ${ }^{22,29}$. The stalled shell thickening, however, shows a notable increase as the cores begin shrinking. This negative correlation between the core areas and shell thicknesses suggests that Li migrates from the core into the shell.

Allowing the once voided particle to further evolve under constant electron-beam irradiation reveals repeated void-forming behavior (Supplementary Movie 5). The particle undergoes further lithiation, filling in the void dynamically formed during core dealloying (Fig. 3a-d). The continued lithiation shows that once the shell-induced mechanical constraint is removed, the core undergoes normal lithiation again. After sufficient expansion and complete filling of the void, the lithiated core repeats the shrinkage and dealloying, nucleating a void in a similar manner (Fig. 3d, e). At this point, the repeated mechanical interaction 

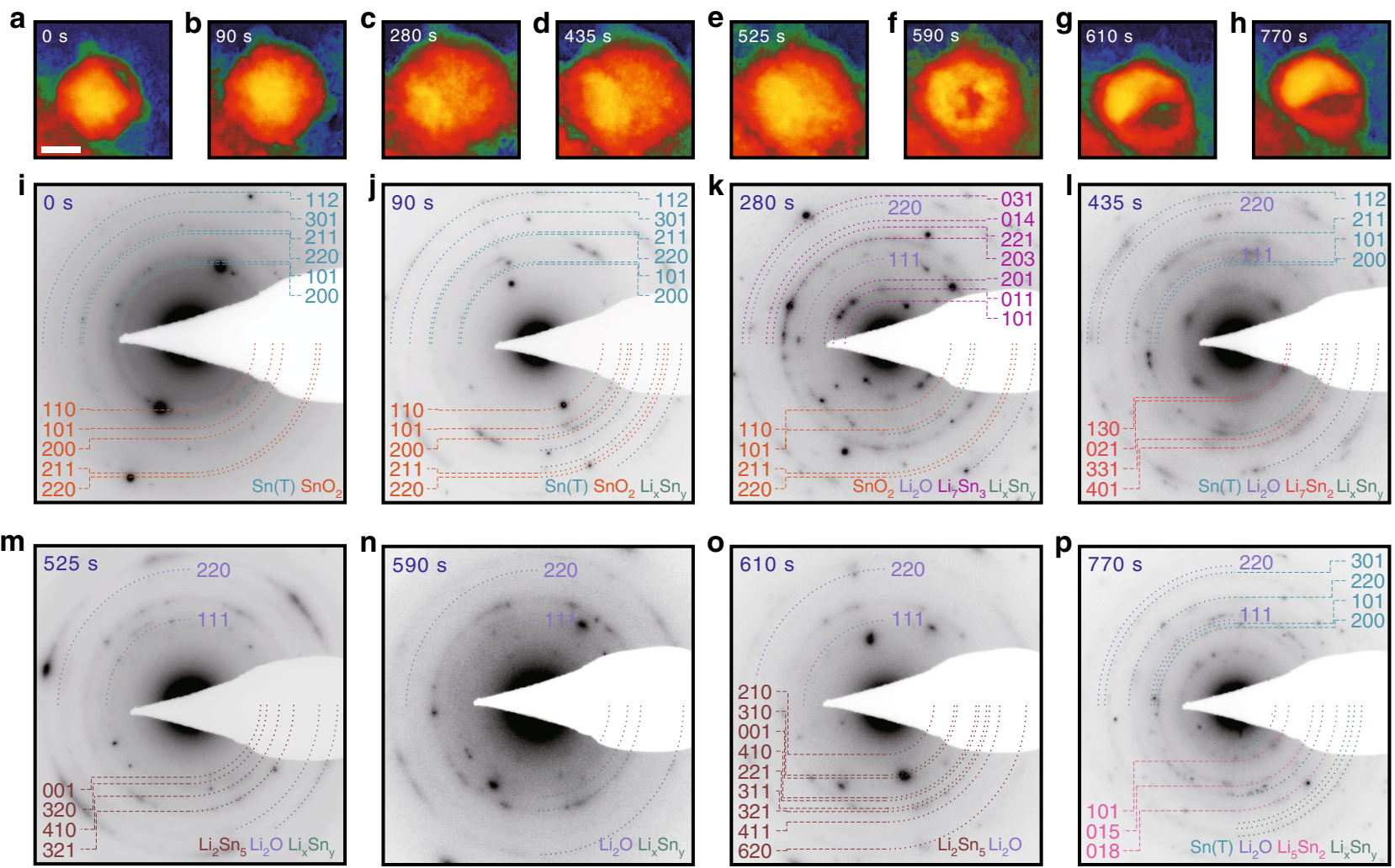

Fig. 1 Lithium (Li)-deficient phases nucleated during lithiation under stress. a-h Lithiation-induced volume changes during in situ lithiation of a $\mathrm{Sn}-\mathrm{SnO} 2$ core-shell particle. i-p The electron diffraction patterns taken at the respective (a-h) moments. The still snapshots are captured from Supplementary Movie 1. The scale bar indicates $100 \mathrm{~nm}$

partially breaks the shell apart, freeing the core from mechanical constraint. The lithiation of the core thereafter continues smoothly without any shrinkage or the formation of voids for an extended time ( $475 \mathrm{~s}$, Fig. $3 \mathrm{e}-\mathrm{h}$ ), similar to our observations of oxide-free Sn nanoparticles (Supplementary Fig. 4). The core expansion is directed towards the region where the shell is partially torn apart (indicated with a red arrow in Fig. 3e), possibly due to the fast $\mathrm{Li}$ transport at this region compared to sluggish $\mathrm{Li}$ transport through the lithiated oxide. It is noted that the core is still in contact with the oxide shell, although not completely covered by the shell. This strongly suggests that the core's dealloying is observed only when the shell completely covers the core surface, that is, when the shell imposes a mechanical constraint on the core. This also directly shows that the dealloying of the core is not driven by the chemical potential difference between the lithiated shell and the core.

Stress-composition coupling in Li alloys. The observed phase and morphological evolution bring our attention to their thermodynamic rationale. Figure 4 illustrates the stress distribution inside the core-shell nanoparticle during lithiation and its effect on the composition. Lithiation-induced volume expansion imposes tensile hoop stress on the shell while imposing compressive stress on the core (Fig. 4a, Supplementary Fig. 11, Supplementary Table 4, and Supplementary Notes 6, 7) ${ }^{30,31}$. This stress contributes to Sn's equilibrium discharge voltage (Supplementary Fig. 12 and Supplementary Note 8$)^{10}$. As particles lithiate from point 1 (Fig. 4b), the shell-imposed stress lowers the core's potential in proportion to the amount of stress (red point 2 in Fig. 4b). At the same time, the Sn islands in the lithiated oxide shell undergo tensile hoop stress and experience elevated potential (blue point 2 in Fig. 4 b). The potential difference between the core and the shell drives Li migration from the core into the shell, causing Kirkendall voids in the former (Supplementary Fig. 13 and Supplementary Note 9). The migration continues until isopotential is reached at around $0.6 \mathrm{~V}$ against $\mathrm{Li}$ (red and blue points 3 in Fig. 4b), where the phases approximately match with the final phases observed in Fig. 1. This whole process is illustrated schematically in Fig. $4 \mathrm{c}$.

The model also suggests that increased stress leads to reduced Li composition. Specifically, thick shells induce a large potential difference by stress and thus result in dealloying at earlier stages of lithiation (Supplementary Figs. 14 and 15, Supplementary Note 10, and Supplementary Movie 6). Performing identical experiments with a small core-to-particle radii ratio ( $a / b$ ratio) indeed results in a reduced maximum $\mathrm{Li}$ composition compared to that with the thin shell (Supplementary Fig. 16). Figure 4d shows a remarkable correlation between the $a / b$ ratio and the maximum volume expansion observed. Interestingly, since the applied hydrostatic stress and volume expansion scale with log $(a / b)$ and the $\mathrm{Li}$ composition, respectively, the achievable $\mathrm{Li}$ composition is proportional to the applied stress.

\section{Discussion}

Considering that the energy scales of electrochemical and mechanical phenomena are in general considered different, the stress-driven core dealloying is surprising. It is noted, however, that the free energy of formation (equivalent to the electrochemical voltage at which the compound forms) of $\mathrm{Li}_{3.5} \mathrm{Sn}$ is only $-0.48 \mathrm{eV}$. In fact, most lithium binary alloys form well under $1 \mathrm{~V}$ against $\mathrm{Li} / \mathrm{Li}^{+}$, with $0.74 \mathrm{~V}$ for $\mathrm{Li}_{2} \mathrm{Sn}_{5}$ being the highest among all lithium tin binary alloys. This energy scale is much smaller than the level at which typical $\mathrm{Li}$-ion batteries with $\mathrm{Co}^{3+/ 4+}$ redox couples operate $(\sim 4 \mathrm{~V})$ since no d-band-based redox couples 

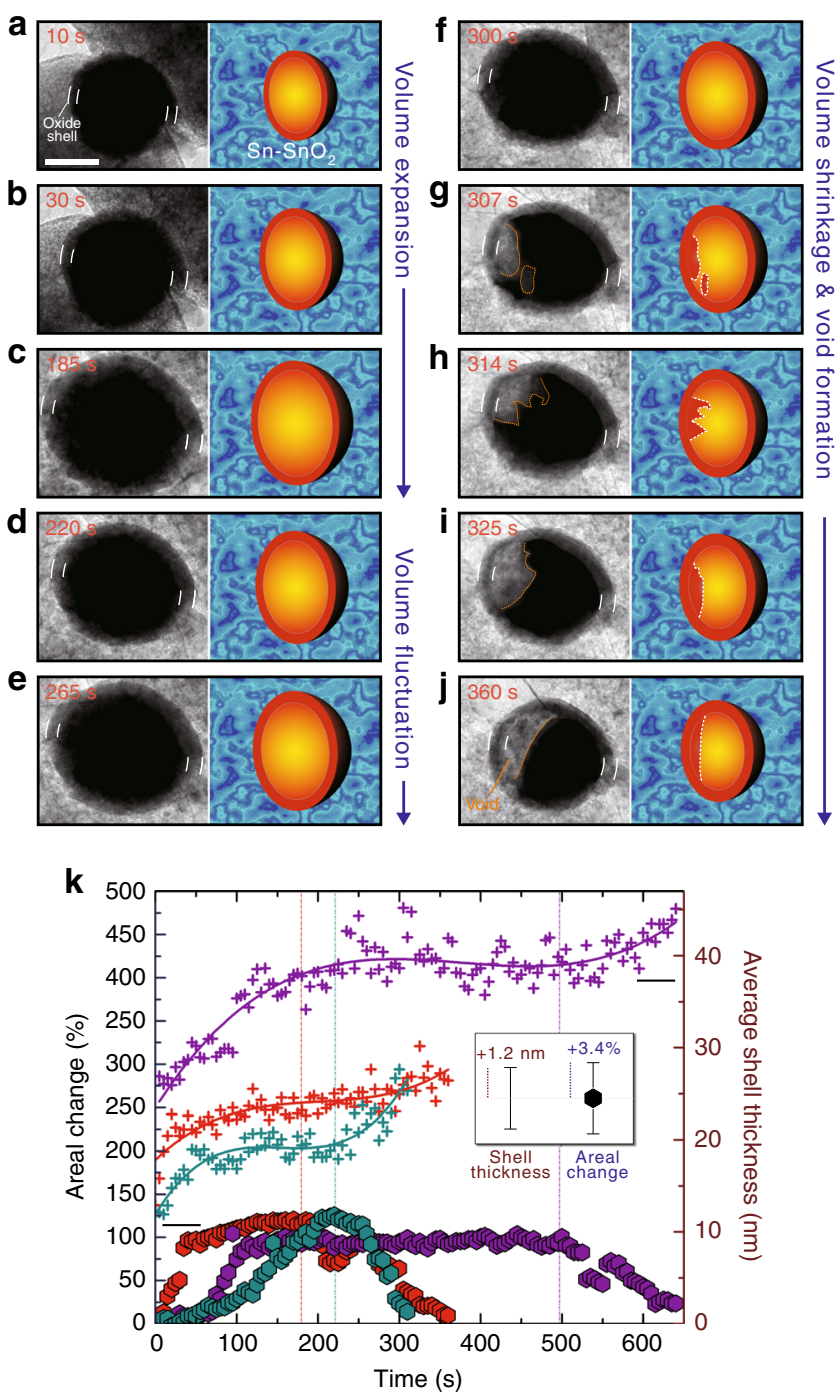

Fig. 2 Void-forming morphological evolution during lithiation under stress. a-j Time-series transmission electron microscopy (TEM) images from Supplementary Movie 3 showing dimensional and morphological evolutions of a $\mathrm{Sn}-\mathrm{SnO}_{2}$ nanoparticle during in situ lithiation for $360 \mathrm{~s}$. White borderlines on each panel note the oxide shell edges. Orange curves highlight the fluctuating void surface on the Sn core. The illustrations next to the TEM images describe the morphological evolution of the particles, including swelling, shrinking, and void formation/evolution. The scale bar indicates $100 \mathrm{~nm}$. k The areal changes (hexagons) and average oxide shell thicknesses (cross-shapes) plotted over the lithiation time, for three similar $\mathrm{Sn}-\mathrm{SnO}_{2}$ particles under two different electron-beam conditions. The error bar indicates the standard deviations obtained from 10 image frames separated by $0.5 \mathrm{~s}$

come into play, but only alloy thermodynamics of the $\mathrm{Li}-\mathrm{Sn}$ system. This energy scale is in fact similar to the energy scales of constraint-induced stress modeled in this work. Supplementary Fig. 14 shows the potential contribution from the mechanical interaction on the shell and the core, assuming a plastically deforming oxide shell. This potential contribution reaches $0.4 \mathrm{~V}$ when the core-to-particle radii ratio falls below 0.8 .

In alloy thermodynamics, stress is well known to affect the alloy composition and phase behavior. During creep tests at $1223 \mathrm{~K}$, nickel-based single crystal SC16 alloy has been reported to form Cr-deficient and Ni-rich $\gamma$ phases due to creep stresses ${ }^{32}$. In eutectoid $\mathrm{Zn}$-Al alloys, metastable $\eta^{\prime}$ phases are reported to undergo a phase transformation due to creep $^{33}$. Since Li possesses high diffusivity at room temperature, the stress-induced $\mathrm{Li}$ composition change and phase transformations can be viewed as a room temperature creep. In this sense, the observations in this work are well aligned with the classical creep phenomena in metallurgy. It is noted that exciting behaviors in stress-induced delithiation on a nanoscale have already been reported and demonstrated. By coating spherical $\mathrm{Si}$ particle surfaces with polypyrrole, Luo et al. ${ }^{34}$ observed that an amorphous $\mathrm{Li}_{x} \mathrm{Si}$ portion within the nanoparticles self-discharged into amorphous $\mathrm{Si}^{34}$. It has also been reported that the constraint-induced mechanical interaction can be a significant factor impeding electrochemical lithiation of silicon ${ }^{30}$.

One may argue that the void formation is in fact phase separation behavior of lithiated tin into $\mathrm{Li}$-rich and $\mathrm{Li}$-poor phases. We note that the elemental mapping of a voided particle exhibits uniform Li distribution and segregated Sn within the particle (Supplementary Fig. 7). The uniform Li distribution first rules out the possibility of the void being a Li-rich phase formed via phase separation. The thickness ratio obtained via electron energy loss spectroscopy (EELS) (Supplementary Fig. 7f, g) further show that the voided area exhibits a sudden decrease in thickness with only approximately $1 / 3$ that of the dealloyed core. The thickness likely corresponds to the outer shell, and strongly asserts for the void formation. The shell, however, does not exhibit notably increased $\mathrm{Li}$ concentration. This may be due to the fact that two-dimensional (2D) elemental mapping is difficult to deconvolute the signals from thick and thin regions; similar $\mathrm{Li}$ concentration from considerably thinner shell suggests increased $\mathrm{Li}$ concentration within the shell than that from the core.

It is noted that the stress model developed above only depends on the $a / b$ ratio, and not on the absolute size of the particle. However, the model also assumes that the shell maintains its constraint against the core, despite the core expansion. Such mechanical integrity can be expected only from nanoscale cores and shells, as large particles may be subject to size-dependent degradation mechanisms. Over the past ten years, we learned that materials become stronger ${ }^{35-37}$ as the material approaches submicroscale. Our observations occur at a scale of $100 \mathrm{~nm}$, where the shell and core are expected to possess nearly ideal strengths.

The stress-composition coupling implies that we can deliberately redistribute $\mathrm{Li}$ according to the applied stress. We demonstrate this by fabricating an electrochemically driven mechanical energy harvester with $\mathrm{Li}_{3} \mathrm{Sn}$ thin films ${ }^{2}$ (Supplementary Fig. 17). The measured short-circuit current corresponds to the amount of redistributed $\mathrm{Li}$ between the identical electrodes due to the different stresses applied by bending. Unbending the device does not generate an electric current in the opposite direction since the two thin films in two-phase equilibria (between $\mathrm{Li}_{5} \mathrm{Sn}_{2}, \mathrm{Li}_{7} \mathrm{Sn}_{2}$ ) maintain a fixed Li chemical potential over a given composition range. This indicates that the current generated is entirely due to the stress gradient and not due to the chemical composition difference. Along with the reported $\mathrm{Li}_{x} \mathrm{Si}$-based harvesters, the present findings suggest that stress-composition coupling is general in Li binary alloys, although the degree of its effect may be modulated by the stress-driven kinetic retardation ${ }^{11}$.

One may also question whether irradiating a liquid cell with an electron beam may cause unexpected side effects. We also seriously considered the similarity and differences between our lithiation technique and the electrochemical conditions. The critical question here is whether the stress-composition coupling we observe is caused by side effects of the electron beam. To ensure scientific rigorousness, we employed multiple characterization techniques including ex situ TEM analyses and in situ SAXS during galvanostatic lithiation. These analyses show the Lideficient phases and voided particle morphology, confirming that the results are reproducible under galvanostatic conditions. In 

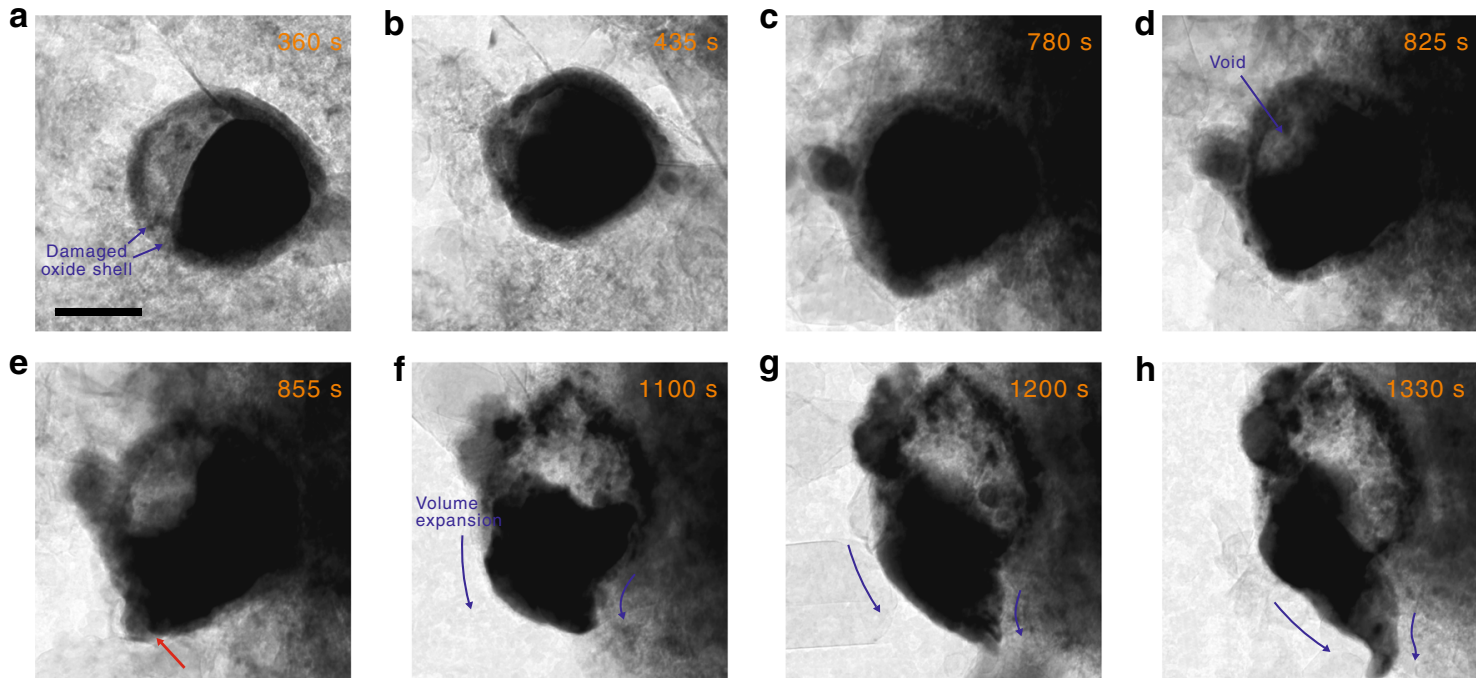

Fig. 3 Time-series images of continued lithiation of a voided particle. a-c The particle repeats lithiation once shell-induced mechanical interaction is released and $\mathbf{d}$ self-discharge again upon sufficient lithiation. e-h As the shell tears apart, however, the core continues normal lithiation despite being partially attached to the shell. The scale bar indicates $100 \mathrm{~nm}$

addition, the sharp contrast between monotonic lithiation of oxide-free Sn nanoparticles and the abnormal lithiation of coreshell nanoparticles under identical GLC condition indicates that stress-driven $\mathrm{Li}$ migration is not caused by electron-beam irradiation.

Despite the foregoing, we employed a commercially available electrolyte solution ( $1 \mathrm{M}$ lithium hexafluorophosphate $\left(\mathrm{LiPF}_{6}\right)$ salt in ethylene carbonate/diethyl carbonate (EC/DEC) mixture) known to be relatively stable against electron-beam irradiation ${ }^{38}$, in efforts to minimize possible electrolyte decomposition (both salt and solvent). We also expected that the conductive graphene layers covering the electrolyte would provide additional protection by radiolytic damage relaxation ${ }^{17,18}$. Under these conditions, control experiments confirm that no visible electrolyte degradation occurs under $7.71 \times 10^{-14} \mathrm{~A} \mathrm{~nm}^{-2}$ electron-beam irradiation, the beam condition employed for the in situ lithiation experiments (Supplementary Fig. 17). The time-series bright-field TEM images show natural liquid fluctuations, yet with no gaseous bubble formation or decomposed particle precipitation.

High-intensity X-ray-based in situ experimental techniques that do not involve any electron-beam damages have recently been developed ${ }^{15,39}$. Nonetheless, these techniques visualize the redox-active transition metal species and may not yet be applied to lithium alloys without transition metal species. In addition, the complex phase and morphological evolution during lithiation of core-shell nanoparticles require nanoscale resolution currently only capable with TEM analyses.

Despite the powerful capabilities of GLC-EM, caution should be exercised because the damage from the electron beam has been reported to be more severe in water, as compared to electrolyte solutions. Schneider et al. ${ }^{40}$ recently showed that a large electronbeam dose might deactivate the etching chemistry of a waterbased solution and erroneously show growth behavior of nanoparticles. The degree of radical formation yield under irradiation has been quantified as $G$ values, and Abellan et al. ${ }^{41}$ showed that the $G$ values for aqueous electrons $\left(\mathrm{e}_{\mathrm{aq}}{ }^{-}\right)$are approximately 20 times higher in magnitude than those of $\mathrm{H}_{2}$ radical formation in toluene. For this reason, electron-beam irradiation to drive redox reactions in organic solvent-based electrolytes has been demonstrated extensively as a successful experimental technique ${ }^{18,42-44}$, while those for aqueous chemistry has been more challenging.
In summary, we directly observe the stress-composition coupling in $\mathrm{Li}$ alloy nanoparticles via GLC-EM. Lithiating a $\mathrm{Sn}-\mathrm{SnO}_{2}$ core-shell nanoparticle results in substantive stress development within the particle, and constraint-induced mechanical stress results in a significant $\mathrm{Li}$ composition change among lithiated core-shell nanoparticles. The strong stress-composition coupling demonstrated in this work has general implications for energy harvesters, sensors, and battery electrode designs. The ability to redistribute a large amount of $\mathrm{Li}$ by applying stress implies immediate applications for mechanical energy harvesters or storage systems. Also, by promoting the coupling effect via engineering the Li diffusivity, we can toughen the electrodes to alleviate their fracture or pulverization during lithiation/delithiation cycles. For instance, the tensile stress field near crack tips will invite nearby Li to fill in and blunt the crack tip, retarding crack propagation ${ }^{7}$. In battery electrode designs, stress can be an active design variable controlling the maximally achievable $\mathrm{Li}$ composition while at the same time preventing electrode fracture.

\section{Methods}

Materials and GLC preparation. Commercially available $\mathrm{Sn}$ ( $\beta$-phase) nanoparticles with a diameter range of $50-200 \mathrm{~nm}$ from Sigma-Aldrich were used. The $\mathrm{Sn}-\mathrm{SnO}_{2}$ core-shell structure was synthesized by thermal oxidation of the $\mathrm{Sn}$ particles under an oxygen environment at $120^{\circ} \mathrm{C}$ for $24 \mathrm{~h}$. The particle maintains strong crystallinity in both its core and shell (Supplementary Fig. 18). Graphene was synthesized on a copper substrate $(0.025$-mm-thick foil, Alfa Aesar) by chemical vapor deposition (Supplementary Fig. 19, Thermal CVD System, Scien Tech Inc.). The Li-ion battery electrolyte is $1 \mathrm{M} \mathrm{LiPF}_{6}$ salt dissolved in ECs and DEC solution mixed at a 1:1 volume ratio.

The GLC is directly fabricated by drop-casting $20 \mu \mathrm{L}$ of Sn nanoparticledispersed electrolyte solution on two overlapped graphene-coated TEM grids (Au 300 mesh, quantifoil R2/2, SPI supplies), followed by suctioning the extra solution ${ }^{17,18}$. By van der Waals attractions between the graphene sheets, a few micron-sized liquid electrolyte pockets containing Sn nanoparticles are formed. After vacuum drying for $24 \mathrm{~h}$, the sample was loaded into a TEM column for in situ experiments. All liquid cell fabrications were conducted in a high-purity argon (Ar)-filled glove box, with moisture $\left(\mathrm{H}_{2} \mathrm{O}\right)$ and oxygen $\left(\mathrm{O}_{2}\right)$ concentrations $<1 \mathrm{ppm}$.

Electrochemical cell tests. For electrochemical tests, Sn working electrodes were prepared by the slurry casting method. The slurry was composed of Sn nanoparticles, carbon black (acetylene, Alfa Aesar), and polyvinylidene fluoride (PVDF, Sigma Aldrich) uniformly mixed at an 8:1:1 mass ratio in a 1-methyl-2-pyrrolidone (Sigma-Aldrich) solution. After depositing the slurry onto a Cu current collector by doctor blading, we dried the slurry for $12 \mathrm{~h}$ in a vacuum chamber $(\mathrm{OV}-12$, Jeio 


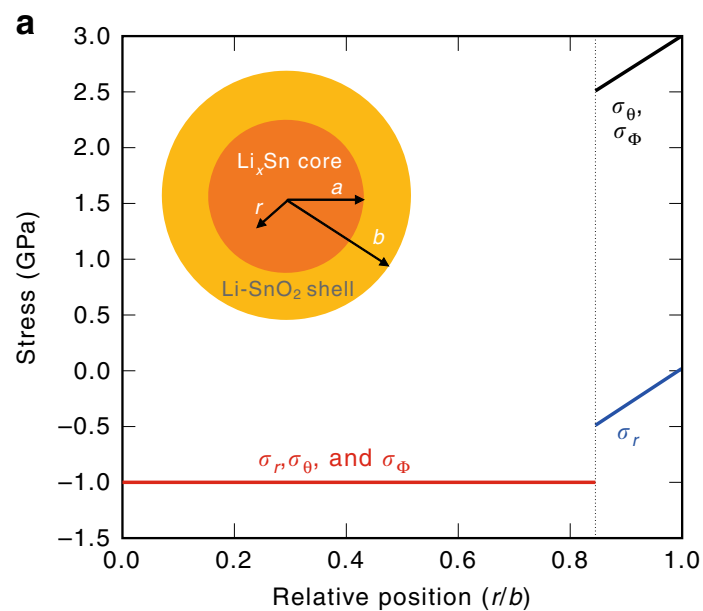

C

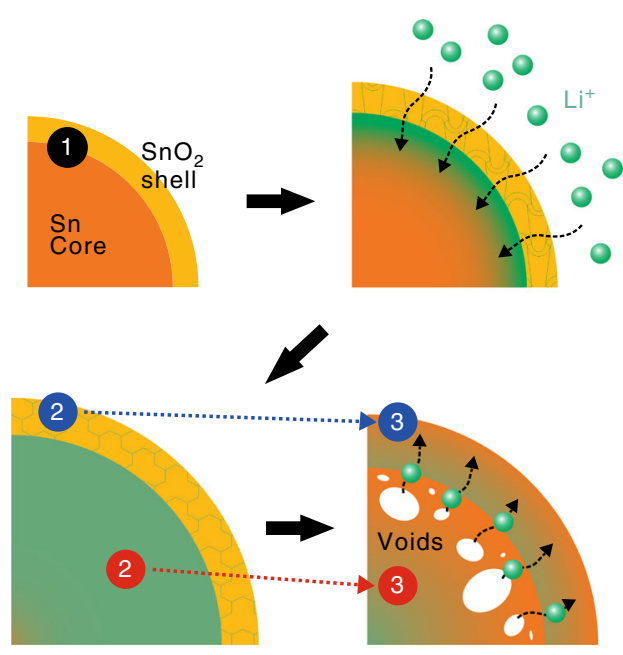

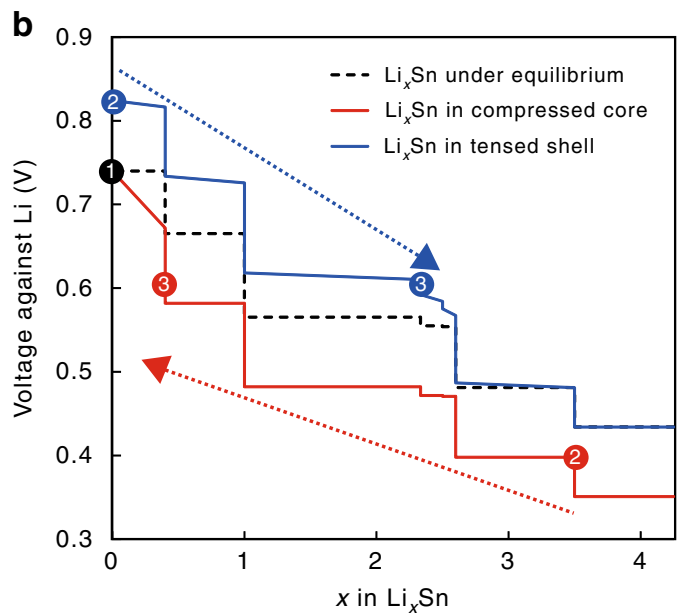

d

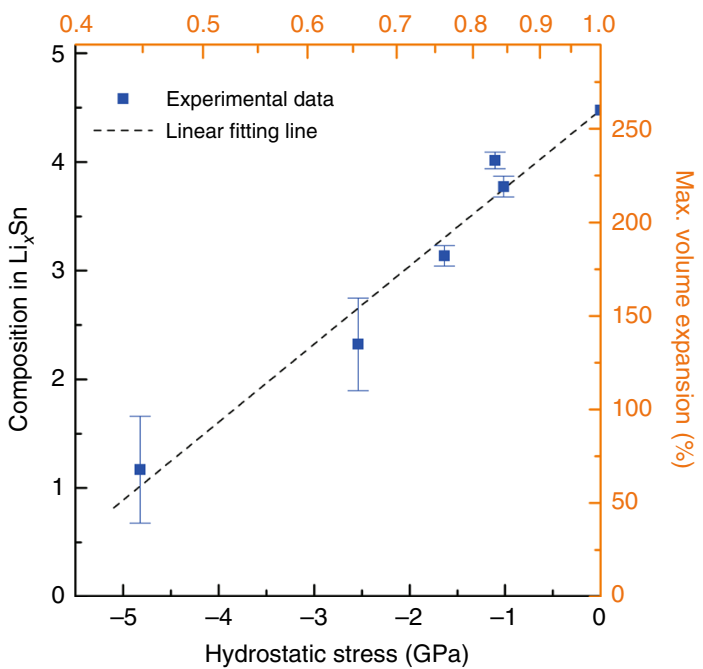

Fig. 4 Thermodynamic rationale for the strong stress-composition coupling. a The stress distribution within the particle during lithiation according to the relative radial position from the particle center, with the inset showing the lithiated particle geometry. The shell undergoes tensile plastic deformation while imposing compressive hydrostatic stress of $1 \mathrm{GPa}$ on the core. $\mathbf{b}, \mathbf{c}$ The stress effect on the equilibrium potential of the Li-Sn alloy system (b) and schematic illustrating the spontaneous core dealloying based on the stress-driven potential differences (c). The numbered circles illustrate the discrete timeframes during lithiation. d The experimentally observed stress-composition coupling in $\mathrm{Li}_{x} \mathrm{Sn}$. The maximum volume expansion observed before dealloying initiation according to the core-to-particle radii ratio $(a / b)$. The error bars indicate standard deviations in volume from 10 image frames separated by $1 \mathrm{~s}$

Tech). A half-cell (ECC-STD, EL-CELL) was assembled with the slurry-based working electrode, Li metal counter electrode, and separator in an argon-filled glove box. $\mathrm{LiPF}_{6}$-based electrolytes were used for the cell assembly, which had an identical composition to that used in the GLC fabrication above. With a cell tester (PARSTAT MC 1000, Princeton Applied Research), charging and discharging measurements were conducted under a constant current condition of a $0.1 \mathrm{C}$-rate at room temperature. The cutoff voltages were 0.05 and $3.0 \mathrm{~V}$. Galvanostatic intermittent titration technique measurements were carried out for both pristine $\mathrm{Sn}$ and core-shell $\mathrm{Sn}-\mathrm{SnO}_{2}$ nanoparticles by periodically applying 1-h duration current pulses at $0.05 \mathrm{C}$, with 5 -h relaxation after each pulse (Supplementary Note 8). The open-circuit voltage at a specific composition was defined by the recovered $\mathrm{Li}$ potential after 5-h rest time and is plotted as a function of the Li composition in Supplementary Fig. 20.

In situ and ex situ TEM characterizations. A JEM-3010 (300 kV, JEOL) microscope with a high-speed charge-coupled device camera (SC200, Gatan) was used to observe the real-time lithiation dynamics of Sn nanoparticles. Supplementary Movies showing the morphological evolutions were recorded with the Gatan Digital Micrograph software (recording rate: 3 frames $\mathrm{s}^{-1}$, Gatan Microscopy Suite). The electron-beam dosage was maintained in a range of $3.0-8.0 \mathrm{~A} \mathrm{~cm}^{-2}$ to initiate the lithiation as well as for TEM imaging. Ex situ TEM observations were carried out with a Tecnai $\mathrm{G}^{2} \mathrm{~F} 30 \mathrm{~S}$-Twin microscope $(300 \mathrm{kV}, \mathrm{FEI})$. After cycling the electrochemical cells, we disassembled the cells under an inert Ar atmosphere. Residual electrolyte on the Sn electrode surfaces was removed by rinsing and sonicating the surface several times with a dimethyl carbonate (DMC) solution. The Sn-dispersed
DMC solution was then drop-casted onto a perforated carbon-coated TEM grid (Cu, 300 Mesh, SPI Supplies). TEM imaging and energy-dispersive spectroscopy elemental analyses were conducted after drying the solvent at room temperature for $12 \mathrm{~h}$. EELS spectra are obtained at $300 \mathrm{kV}$ accelerating voltage (Supplementary Fig. 21 and Supplementary Note 2)

In situ SAXS measurements. The crystalline phase evolution during lithiation of $\mathrm{Sn}-\mathrm{SnO}_{2}$ particles was tracked via SAXS (D/MAX-2500 with R-AXIS IV++ diffractometer, Rigaku) (Supplementary Fig. 3). The X-ray beam $\left(\mathrm{Cu} \mathrm{K} \mathrm{K}_{\alpha}\right.$ ) was generated by electron-beam irradiation on a rotating anode type $\mathrm{Cu}$ plate at $50 \mathrm{kV}$ and $100 \mathrm{~mA}$. The X-ray beam filtered with a 0.15 -mm-diameter slit was collimated into the assembled coin cell. To allow both the incident and scattered beam to pass through the lithiated $\mathrm{Sn}-\mathrm{SnO}_{2}$ particle electrode, both sides of the coin cell case have X-ray transparent polymer windows. Supplementary Fig. 22 schematically illustrates the configuration of our in situ SAXS experiment setup and shows the actual device setup. For the electrochemical cycling test, the coin cell was assembled with a Li foil counter electrode and discharged under a constant current condition $(0.05 \mathrm{C})$. The data acquisition was carried out at 1-h intervals. Using RigakuDisplay software, we processed the obtained time-series 2D intensity maps into time-series $1 \mathrm{D}$ spectra by summing the intensity for all radial directions as a function of the scattering angle, ranging from $15^{\circ}$ to $50^{\circ}$.

Ex situ XRD measurements. A high-resolution powder X-ray diffractometer (SmartLab, $45 \mathrm{kV} 200 \mathrm{~mA}$, Rigaku) was used for crystalline phase analyses in the 
cycled $\mathrm{Sn}-\mathrm{SnO}_{2}$ nanoparticles. After cycling, we disassembled the coin cell in an Ar-filled glove box and scraped the active materials off a $\mathrm{Cu}$ current collector. The active materials were then loaded into a home-made air-tight holder with an X-ray transparent Kapton polyimide (PI) film window, avoiding air exposure during measurements. Under the normal $\theta / 2 \theta$ scan mode, the $2 \theta$ scan range, scan speed, and step size were set to be $20-35^{\circ}, 4^{\circ} \mathrm{min}^{-1}$, and $0.01^{\circ}$, respectively.

Ab initio calculations. All density functional theory calculations were performed using Vienna ab initio simulations package ${ }^{45}$. The exchange correlation functional was treated via generalized-gradient approximation ${ }^{46}$ and projector-augmented wave method implemented with Perdew-Burke-Erzenhof functional were used ${ }^{47}$. Both ionic positions and cell parameters were allowed to fully relax during the calculations, and an energy cutoff of $520 \mathrm{eV}$ was used. K-point grid density of at least 1000 per atom was used, and spin-polarized calculations were performed with no Hubbard-like U correction.

Energy harvester fabrication. The energy harvesters were fabricated on $25 \mu \mathrm{m}$ PI substrates, with 100- and 15-nm-thick current collector layers of $\mathrm{Pt}$ and $\mathrm{Ti}$, respectively. Sn electrodes were then deposited up to $100 \mathrm{~nm}$, at a deposition rate of $0.2 \AA \mathrm{s}^{-1}$. All depositions were performed by sputtering target metals onto the substrates. The $\mathrm{Sn}$ thin films were electrochemically lithiated up to $\mathrm{Li}_{3} \mathrm{Sn}$ composition and were assembled into an energy harvester by cutting the thin film into half, sandwiching a layer of commercial Li electrolyte (EC:DEC 1:1 volume ratio, $1 \mathrm{M} \mathrm{LiPF}_{6}$ ), and packaging with an Al-covered pouch cell. The short-circuit current was measured with a Keithley 6485 picoammeter, while the device was being bent via an Arduino-controlled servo motor with $8 \mathrm{~mm}$ radius of curvature.

\section{Data availability}

All data supporting the conclusions of this study are included in the manuscript and its Supplementary materials.

Received: 28 November 2018 Accepted: 2 July 2019

Published online: 31 July 2019

\section{References}

1. Lee, S. W. et al. Kinetics and fracture resistance of lithiated silicon nanostructure pairs controlled by their mechanical interaction. Nat. Commun. 6, 7533 (2015).

2. Kim, S. et al. Electrochemically driven mechanical energy harvesting. Nat. Commun. 7, 10146 (2016).

3. $\mathrm{Bu}, \mathrm{L}$. et al. Biaxially strained $\mathrm{PtPb} / \mathrm{Pt}$ core/shell nanoplate boosts oxygen reduction catalysis. Science 354, 1410-1414 (2016).

4. Strasser, P. et al. Lattice-strain control of the activity in dealloyed core-shell fuel cell catalysts. Nat. Chem. 2, 454 (2010).

5. Davis, D. A. et al. Force-induced activation of covalent bonds in mechanoresponsive polymeric materials. Nature 459, 68 (2009).

6. Obrovac, M. N. \& Chevrier, V. L. Alloy negative electrodes for Li-ion batteries. Chem. Rev. 114, 11444-11502 (2014).

7. Wang, X. et al. High damage tolerance of electrochemically lithiated silicon. Nat. Commun. 6, 8417 (2015).

8. Zhang, S., Zhao, K., Zhu, T. \& Li, J. Electrochemomechanical degradation of high-capacity battery electrode materials. Prog. Mater. Sci. 89, 479-521 (2017).

9. $\mathrm{Gu}, \mathrm{M}$. et al. Bending-induced symmetry breaking of lithiation in germanium nanowires. Nano Lett. 14, 4622-4627 (2014).

10. Cui, Z., Gao, F. \& Qu, J. A finite deformation stress-dependent chemical potential and its applications to lithium ion batteries. J. Mech. Phys. Solids 60, 1280-1295 (2012).

11. Liu, X. H. et al. Self-limiting lithiation in silicon nanowires. ACS Nano 7, 1495-1503 (2013).

12. Hwang, S. et al. Strain coupling of conversion-type $\mathrm{Fe}_{3} \mathrm{O}_{4}$ thin films for lithium ion batteries. Angew. Chem. Int. Ed. 56, 7813-7816 (2017).

13. Larché, F. C. \& Cahn, J. W. Overview no. 41 The interactions of composition and stress in crystalline solids. Acta Metall. 33, 331-357 (1985).

14. Ebner, M., Marone, F., Stampanoni, M. \& Wood, V. Visualization and quantification of electrochemical and mechanical degradation in Li ion batteries. Science 342, 716-720 (2013).

15. Lim, J. et al. Origin and hysteresis of lithium compositional spatiodynamics within battery primary particles. Science 353, 566-571 (2016).

16. de Jonge, N. \& Ross, F. M. Electron microscopy of specimens in liquid. Nat. Nanotechnol. 6, 695-704 (2011).

17. Yuk, J. M. et al. High-resolution EM of colloidal nanocrystal growth using graphene liquid cells. Science 336, 61-64 (2012).
18. Yuk, J. M., Seo, H. K., Choi, J. W. \& Lee, J. Y. Anisotropic lithiation onset in silicon nanoparticle anode revealed by in situ graphene liquid cell electron microscopy. ACS Nano 8, 7478-7485 (2014).

19. Wang, J. \& Huggins, R. A. Behavior of some binary lithium alloys as negative electrodes in organic solvent-based electrolytes. J. Electrochem. Soc. 133 457-460 (1986).

20. Huang, J. Y. et al. In situ observation of the electrochemical lithiation of a single $\mathrm{SnO}_{2}$ nanowire electrode. Science 330, 1515-1520 (2010).

21. Meduri, P., Clark, E., Dayalan, E., Sumanasekera, G. U. \& Sunkara, M. K. Kinetically limited de-lithiation behavior of nanoscale tin-covered tin oxide nanowires. Energ. Environ. Sci. 4, 1695-1699 (2011).

22. Nie, A. et al. Twin boundary-assisted lithium-ion transport. Nano Lett. 15 610-615 (2015).

23. Ichitsubo, T. et al. Mechanical-energy influences to electrochemical phenomena in lithium-ion batteries. J. Mater. Chem. 21, 2701-2708 (2011).

24. Chen, Q. \& Sieradzki, K. Spontaneous evolution of bicontinuous nanostructures in dealloyed Li-based systems. Nat. Mater. 12, 1102-1106 (2013).

25. Sun, J. et al. Liquid-like pseudoelasticity of sub-10-nm crystalline silver particles. Nat. Mater. 13, 1007 (2014).

26. Cao, K. et al. In situ TEM investigation on ultrafast reversible lithiation and delithiation cycling of Sn@C yolk-shell nanoparticles as anodes for lithium ion batteries. Nano Energy 40, 187-194 (2017).

27. Obrovac, M. N., Christensen, L., Le, D. B. \& Dahn, J. R. Alloy design for lithium-ion battery anodes. J. Electrochem. Soc. 154, A849-A855 (2007).

28. Wang, J. et al. Structural evolution and pulverization of tin nanoparticles during lithiation-delithiation cycling. J. Electrochem. Soc. 161, F3019-F3024 (2014).

29. Li, Q. et al. In situ TEM on the reversibility of nanosized Sn anodes during the electrochemical reaction. Chem. Mater. 26, 4102-4108 (2014).

30. Zhao, K. et al. Concurrent reaction and plasticity during initial lithiation of crystalline silicon in lithium-ion batteries. J. Electrochem. Soc. 159, A238-A243 (2012).

31. Verbrugge, M. W., Qi, Y., Baker, D. R. \& Cheng, Y.-T. in Advances in Electrochemical Science and Engineering Ch. 6 (eds Alkire, R. C., Bartlett, P. N. \& Lipkowski, J.) 193-226 (Wiley-VCH Verlag, Weinheim, 2015).

32. Zhang, Y., Wanderka, N., Schumacher, G., Schneider, R. \& Neumann, W. Phase chemistry of the superalloy SC16 after creep deformation. Acta Mater. 48, 2787-2793 (2000).

33. Zhu, Y. H., Orozco, E. \& Murphy, S. Creep-induced phase transformations in furnace cooled $\mathrm{Zn}-\mathrm{Al}$ alloy. J. Mater. Sci. 32, 5811-5815 (1997).

34. Luo, L. et al. Surface coating constraint induced self-discharging of silicon nanoparticles as anodes for lithium ion batteries. Nano Lett. 15, 7016-7022 (2015).

35. Shan, Z. W., Mishra, R. K., Syed Asif, S. A., Warren, O. L. \& Minor, A. M. Mechanical annealing and source-limited deformation in submicrometrediameter Ni crystals. Nat. Mater. 7, 115-119 (2008).

36. Liu, X. H. et al. Size-dependent fracture of silicon nanoparticles during lithiation. ACS Nano 6, 1522-1531 (2012).

37. Chan, C. K. et al. High-performance lithium battery anodes using silicon nanowires. Nat. Nanotechnol. 3, 31-35 (2008).

38. Abellan, P. et al. Probing the degradation mechanisms in electrolyte solutions for Li-ion batteries by in situ transmission electron microscopy. Nano Lett. 14, 1293-1299 (2014)

39. Li, Y. et al. Dichotomy in the lithiation pathway of ellipsoidal and platelet $\mathrm{LiFePO}_{4}$ particles revealed through nanoscale operando state-of-charge imaging. Adv. Funct. Mater. 25, 3677-3687 (2015).

40. Schneider, N. M. et al. Electron-water interactions and implications for liquid cell electron microscopy. J. Phys. Chem. C. 118, 22373-22382 (2014).

41. Abellan, P. et al. Gaining control over radiolytic synthesis of uniform sub-3 nanometer palladium nanoparticles: Use of aromatic liquids in the electron microscope. Langmuir 32, 1468-1477 (2016).

42. Zheng, H. et al. Observation of single colloidal platinum nanocrystal growth trajectories. Science 324, 1309-1312 (2009).

43. Cheong, J. Y. et al. Growth dynamics of solid electrolyte interphase layer on $\mathrm{SnO}_{2}$ nanotubes realized by graphene liquid cell electron microscopy. Nano Energy 25, 154-160 (2016).

44. Hwa, Y., Seo, H. K., Yuk, J. M. \& Cairns, E. J. Freeze-dried sulfur-graphene oxide-carbon nanotube nanocomposite for high sulfur-loading lithium/sulfur cells. Nano Lett. 17, 7086-7094 (2017).

45. Kresse, G. \& Furthmüller, J. Efficient iterative schemes for $a b$ initio totalenergy calculations using a plane-wave basis set. Phys. Rev. B 54, 11169-11186 (1996).

46. Perdew, J. P., Burke, K. \& Ernzerhof, M. Generalized gradient approximation made simple. Phys. Rev. Lett. 77, 3865-3868 (1996).

47. Blöchl, P. E. Projector augmented-wave method. Phys. Rev. B 50, 17953-17979 (1994). 


\section{Acknowledgements}

H.K.S., J.Y.P., and J.H.C. thank M.-J.W. in XRD Lab of KAIST Analysis Center for Research Advancement (KARA) for technical support with in situ SAXS experiments. This work was conducted as part of the KAIST-funded Global Singularity Research Program for 2019 and supported by the Nano Material Technology Development Program through the National Research Foundation of Korea (NRF) funded by the Ministry of Science, ICT and Future Planning (2009-0082580), which provided support for TEM analyses and sample preparation; a National Research Foundation of Korea (NRF) grant funded by the Korean government (MSIP; Ministry of Science, ICT \& Future Planning) (NRF-2018R1C1B6002624), which provided support for graphene growth and TEM sample preparation; and a National Research Foundation of Korea (NRF) grant funded by the Korean government (MSIP; Ministry of Science, ICT \& Future Planning) (NRF2018M3A7B4065625), which provided support for preparation of electrode materials and electrochemical measurement. S.K. and C.-Y.K. acknowledge support from a National Research Council of Science and Technology (NST) grant by the Korean government (MSIP) (No. CAP-17-04-KRISS); Korea Institute of Science and Technology institutional programs (2E29400, 2E28990); computational resources from KISTI Supercomputing Centers through KSC-2018-S1-0018; and a support from the Energy Technology Development Project (KETEP) grant funded by the Ministry of Trade, Industry and Energy, Republic of Korea (Project no. 2018201010636 A). J.Y.P. specially thanks the NRF funded by the Korean government (MSIP; Ministry of Science, ICT \& Future Planning) for scholarship support through the Global Ph.D. Fellowship Program (NRF2018H1A2A1060105).

\section{Author contributions}

H.K.S., S.K. and J.M.Y. designed and performed in situ and ex situ TEM experiments. H.K.S. and J.M.Y. prepared all samples for the experiments and H.K.S, J.Y.P., J.H.C., K.S. D., and S.-S.K. conducted electrochemical cell measurements and data analyses. S.K.,

C.-Y.K. and K.Z. performed thermodynamic calculations and plasticity modelling in this study. S.K. and M.-S. N. fabricated the energy harvester. H.K.S., S.K. and J.M.Y. wrote the manuscript. All authors participated in the analysis and discussion of the results under the supervision of S.K. and J.M.Y.

\section{Additional information}

Supplementary Information accompanies this paper at https://doi.org/10.1038/s41467019-11361-z.

Competing interests: The authors declare no competing interests.

Reprints and permission information is available online at http://npg.nature.com/ reprintsandpermissions/

Peer review information: Nature Communications thanks Sulin Zhang and other anonymous reviewer(s) for their contribution to the peer review of this work.

Publisher's note: Springer Nature remains neutral with regard to jurisdictional claims in published maps and institutional affiliations.

\section{(c) (1)}

Open Access This article is licensed under a Creative Commons Attribution 4.0 International License, which permits use, sharing, adaptation, distribution and reproduction in any medium or format, as long as you give appropriate credit to the original author(s) and the source, provide a link to the Creative Commons license, and indicate if changes were made. The images or other third party material in this article are included in the article's Creative Commons license, unless indicated otherwise in a credit line to the material. If material is not included in the article's Creative Commons license and your intended use is not permitted by statutory regulation or exceeds the permitted use, you will need to obtain permission directly from the copyright holder. To view a copy of this license, visit http://creativecommons.org/ licenses/by/4.0/.

(C) The Author(s) 2019 